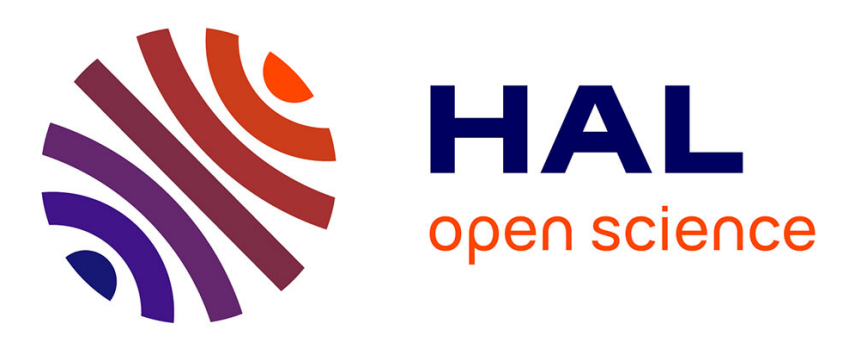

\title{
Anomalous thermal conductivity enhancement in low dimensional resonant nanostructures due to imperfections
}

Hongying Wang, Yajuan Cheng, Zheyong Fan, Yangyu Guo, Zhongwei Zhang, Marc Bescond, Massahiro Nomura, Tapio Ala-Nissila, Sebastian Volz, Shiyun Xiong

\section{To cite this version:}

Hongying Wang, Yajuan Cheng, Zheyong Fan, Yangyu Guo, Zhongwei Zhang, et al.. Anomalous thermal conductivity enhancement in low dimensional resonant nanostructures due to imperfections. Nanoscale, 2021, 13 (22), pp.10010-10015. 10.1039/D1NR01679B . hal-03406379

\section{HAL Id: hal-03406379 \\ https://hal.science/hal-03406379}

Submitted on 28 Oct 2021

HAL is a multi-disciplinary open access archive for the deposit and dissemination of scientific research documents, whether they are published or not. The documents may come from teaching and research institutions in France or abroad, or from public or private research centers.
L'archive ouverte pluridisciplinaire HAL, est destinée au dépôt et à la diffusion de documents scientifiques de niveau recherche, publiés ou non, émanant des établissements d'enseignement et de recherche français ou étrangers, des laboratoires publics ou privés. 


\title{
Anomalous thermal conductivity enhancement in low dimensional resonant nanostructures due to imperfections
}

\author{
Hongying Wang $^{1}$, Zheyong Fan ${ }^{2}$, Yajuan Cheng ${ }^{3}$, Yangyu Guo ${ }^{4}$, Zhongwei Zhang ${ }^{4}$, Marc \\ Bescond $^{6}$, Massahiro Nomura ${ }^{4}$, Tapio Ala-Nissila ${ }^{2,5}$, Sebastian Volz ${ }^{6}$, and Shiyun Xiong ${ }^{1,4 *}$ \\ 1 Institute of Functional Nano and Soft Materials (FUNSOM), \\ Jiangsu Key Laboratory for Carbon-Based Functional Materials and Devices, \\ Soochow University, 199 Ren'ai Road, Suzhou, 215123, Jiangsu, P. R. China. \\ 2 MSP group, QTF Centre of Excellence, Department of Applied Physics, Aalto University, FI-00076 Aalto, Finland \\ 4 Institute of Industrial Science, The University of Tokyo, Tokyo 153-8505, Japan. \\ 3 Key Laboratory of Organic Synthesis of Jiangsu Province and the State and \\ Local Joint Engineering Laboratory for Novel Functional Polymeric Materials, \\ College of Chemistry, Chemical Engineering and Materials Science, \\ Soochow University, Suzhou 215123, P. R. China. \\ 5 Interdisciplinary Centre for Mathematical Modelling, \\ Department of Mathematical Sciences, Loughborough University, \\ Loughborough, Leicestershire LE11 3TU, UK. \\ 6 Laboratory for Integrated Micro Mechatronic Systems (LIMMS/CNRS-IIS), \\ The University of Tokyo, Tokyo 153-8505, Japan.
}

\section{Abstract}

Nanophononic metamaterials have broad applications in fields such as heat management, thermoelectric energy conversion, and nanoelectronics. Phonon resonance in pillared low-dimensional structures has been suggested to be a feasible approach to reduce thermal conductivity (TC). In this work, we study the effects of imperfections in pillared nanostructures based on graphene nanoribbons (GNR), using classical molecular dynamics simulations and harmonic lattice dynamics. The TC of perfect pillared GNR is only about $13 \%$ of that of pristine GNR due to the strong phonon resonant hybridization in pillared GNR. However, introducing imperfections such as vacancy defects and mass mismatch between the pillars and the base material, and alloy disorder in the pillars, can weaken the resonant hybridization and abnormally increase the TC. We show that both vacancy defects and mass mismatch can reduce the penetration of the resonant modes from the pillars into the base material, while the alloy disorder in the pillars can scatter the phonons inside them, which turns regular resonance into a random one with weaker hybridization. Our work provides useful insight into the phonon resonance mechanisms in experimentally relevant low dimensional nanostructures containing various imperfections.

\section{INTRODUCTION}

Due to the ability of tuning low frequency phonon transport, nanophononic metamaterials based on phonon resonance have received great interests [1-10] since it was proposed by Davis and Hussein [11]. Different from the phonon scattering mechanism, which is based on the phonon particle picture and relies on the inclusion of scattering sources inside a material, the phonon resonance mechanism is based on the phonon wave effect and relies on the design of surface resonant structures on top of a base material, which is usually a wire or a film. The incoming and outgoing waves in the surface resonant structures can interfere with each other to form standing waves similar to a closed organ-pipe mode. The standing waves (resonant modes) from the surface resonant structures can hybridize (couple) with the propagating waves in the base material through an anti-crossing effect, which can greatly reduce both the group velocities and the relaxation times of the phonons in the base material $[3,12]$. Through a proper design of the surface resonant struc-

* xiongshiyun216@163.com tures, the transport of phonons in the base material with relatively long wavelengths can be efficiently engineered $[3,13,14]$, which is complementary to the phonon scattering mechanism that mainly affects the transport of phonons with relatively short wavelengths [13]. Because the resonant structures are located on the surface of a base material, their effects on electron transport in the base material are expected to be limited, which could be beneficial for thermoelectric applications [15-17].

While it is well known that phonon resonance can be controlled by the geometrical parameters (such as pillar height and period) of the resonant structures [3], it remains unclear how it can be affected or tuned by imperfections. In this work, we carry out a systematic study on this question by employing classical molecular dynamics (MD) simulations and harmonic lattice dynamics (LD) calculations, taking pillared graphene nanoribbons (GNR) as a prototypical material. We find that the introduction of imperfections in the pillars and the interfaces between the pillars and the base material can effectively reduce the resonant coupling between the resonant and propagating modes and abnormally enhance the TC. This finding suggests that the quality of the pillars and the interfaces are as important as the geometrical 
parameters for tuning the phonon resonance in pillared low-dimensional nanostructures.

\section{MODELS}

For simplicity, we only consider zigzag GNR, i.e., GNR with edges along the zigzag direction; see Fig. 1(a). The width of the GNR is chosen as $L_{\mathrm{NR}}=4.1 \mathrm{~nm}$. Starting from this point, we constructed the various resonant structures with the basic building blocks shown in Figs. $1(\mathrm{~b})-(\mathrm{e})$. (a)

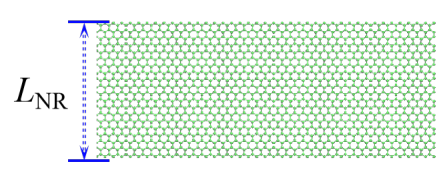

(c)

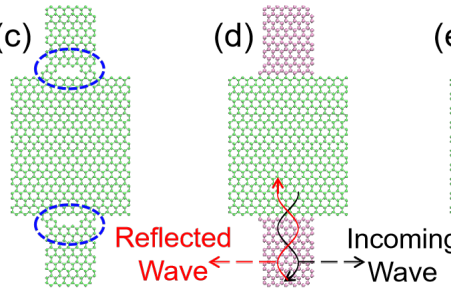

(b)

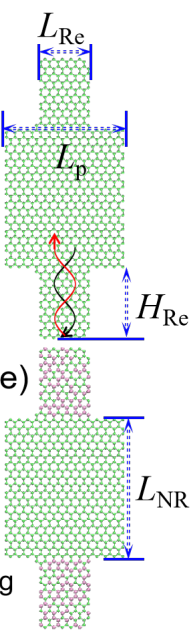

FIG. 1. Schematic illustration of (a) the pristine GNR, (b) the PR-GNR, (c) the DR-GNR, (d) the HR-GNR, and (e) the AR-GNR. The purple color in (d) and (e) indicates atoms with masses different from those of the base graphene flake, see text for details. All the structures are finite in the vertical direction, but are supposed to repeat infinitely in the horizontal direction. In practice, using the length of about $120 \mathrm{~nm}$ in the horizontal direction (with 15 periods for each resonant structure) and periodic boundary conditions ensures the convergence of the properties relevant to our study.

The pillars in these resonant structures have a height of $H_{\operatorname{Re}}=10.2 \mathrm{~nm}$ and a width of $L_{\operatorname{Re}}=4.2 \mathrm{~nm}$, and the period of the resonant structures is $L_{\mathrm{p}}=8.1 \mathrm{~nm}$; see Fig. 1(b) for an illustration of these geometrical parameters. In Fig. 1(b), the pillars are extra graphene flakes attached to the two edges of the middle GNR, and we call the resulting resonant structure the perfect resonant GNR (PR-GNR). The resonant structure in Fig. 1(c) is called the defective resonant GNR (DR-GNR), which is obtained from the PR-GNR by creating vacancy defects (of size $3.2 \times 0.4 \mathrm{~nm}^{2}$ ) around the interface between the pillars and the middle GNR. The resonant structure in Fig. 1(d) is called the heterogeneous resonant GNR (HRGNR), which is obtained from the PR-GNR by changing the mass of the atoms in the pillars. We consider a mass ratio of 2 and a mass ratio of 4 between the pillars' and base material's atoms and call the corresponding structures HR-GNR-2m and HR-GNR-4m, respectively. The resonant structure in Fig. 1(e) is called the alloyed resonant GNR (AR-GNR), which is obtained from the PRGNR by doubling the mass of (uniformly randomly chosen) half of the atoms in the pillars. Last, by combining the DR-GNR and AR-GNR models, we obtain a resonant structure called the defective alloyed resonant GNR (DAR-GNR).

\section{COMPUTATIONAL METHODS}

MD simulations were performed using the GPUMD package $[18,19]$ with the optimized Tersoff potential [20] and a time step of $0.5 \mathrm{fs}$. All simulations were performed at a target temperature of $300 \mathrm{~K}$. We used the homogeneous nonequilibrium MD (HNMED) method [21, 22] to calculate the TC. The running TC as a function of the production time $t$ is evaluated as [22]:

$$
\kappa(t)=\frac{1}{t} \int_{0}^{t} d \tau \frac{\left\langle J_{q}(\tau)\right\rangle_{\mathrm{ne}}}{T V F_{\mathrm{e}}},
$$

where $V$ refers to the system volume, $T$ to the system temperature, $F_{\mathrm{e}}$ is the driving force parameter, and $\left\langle J_{q}(\tau)\right\rangle_{\text {ne }}$ the nonequilibrium heat current induced by the driving force.

In the HNEMD simulations, the TC can be spectrally decomposed in the frequency domain as [22-26]:

$$
\kappa(\omega)=\frac{2 \widetilde{K}(\omega)}{T V F_{\mathrm{e}}} .
$$

Here, $\widetilde{K}(\omega)$ is the Fourier transform of the virial-velocity time correlation function defined as [26]

$$
K(t)=\sum_{i}\left\langle\mathbf{W}_{i}(0) \cdot \boldsymbol{v}_{i}(t)\right\rangle,
$$

where $\mathbf{W}_{i}$ represents the virial tensor for atom $i$ as defined in Ref. 26 and $\boldsymbol{v}_{i}$ is the velocity of atom $i$.

From $\kappa(\omega)$ and the spectral ballistic conductance $G(\omega)$ obtained from an NEMD simulation with a short conducting channel $[23,24,27]$, the spectral phonon mean free path $(\mathrm{MFP}) \lambda(\omega)$ can be calculated as [22]:

$$
\lambda(\omega)=\kappa(\omega) / G(\omega) .
$$

\section{RESULTS}

Figures 2(a) and 2(b) show the TC as a function of the production time in the HNEMD simulations for pristine GNR and PR-GNR, respectively. For pristine GNR, we considered the driving force parameters from $F_{\mathrm{e}}=0.04$ $\mu \mathrm{m}^{-1}$ to $F_{\mathrm{e}}=0.1 \mu \mathrm{m}^{-1}$; for PR-GNR, we considered $F_{\mathrm{e}}=0.3 \mu \mathrm{m}^{-1}$ to $F_{\mathrm{e}}=0.5 \mu \mathrm{m}^{-1}$. Within these ranges of $F_{\mathrm{e}}$, there is no systematic dependence of TC on $F_{\mathrm{e}}$, suggesting that linear response is achieved in the HNEMD 

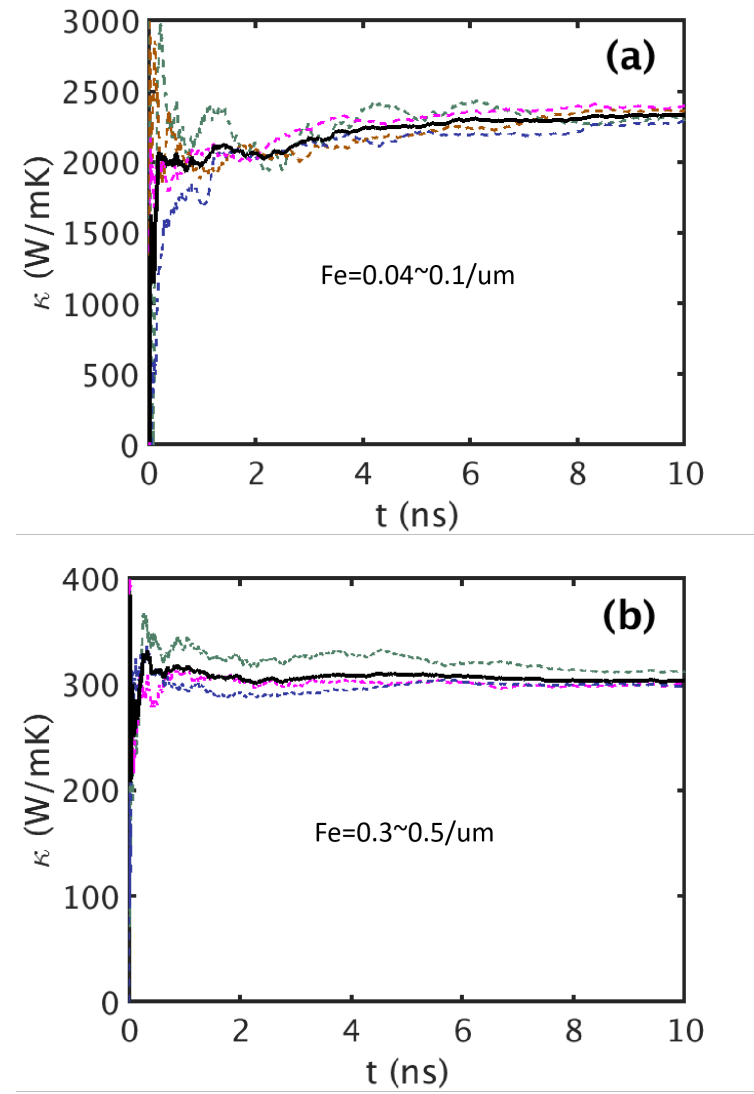

FIG. 2. Running TC $\kappa$ of (a) GNR and (b) PR-GNR as a function of the production time $t$ in HNEMD simulations. The range of magnitudes of the driving force parameter $F_{\mathrm{e}}$ are indicated. The dashed lines represent individual runs and the solid lines represent their averages.

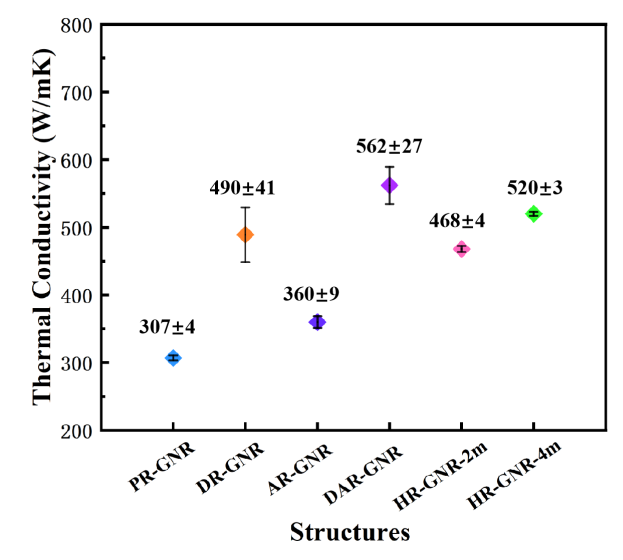

FIG. 3. Converged TC for the various structures considered here (for abbreviations see Fig. 1).

simulations. We adopted the block-averaging method [22] to obtain an average TC and an error estimate for each system: $\kappa=2208 \pm 19 \mathrm{~W} / \mathrm{mK}$ for pristine GNR and $\kappa=307 \pm 4 \mathrm{~W} / \mathrm{mK}$ for PR-GNR. The TC of the GNR

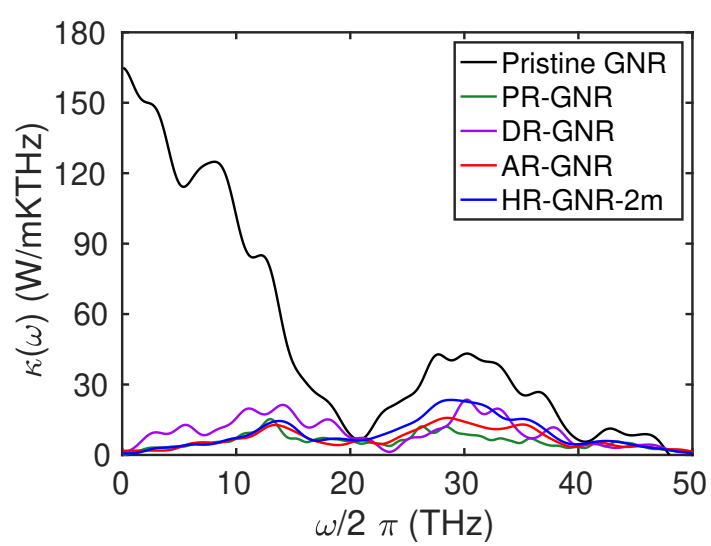

FIG. 4. Spectral thermal conductivity for the various structures considered in this paper, calculated according to Eq. (2).

is only about $20 \%$ smaller than that of two-dimensional graphene [22], which suggests that the phonon-boundary scatterings caused by the edges in the GNR are rather specular and have small effects on the TC. In contrast, by attaching resonant pillars to the GNR to form the PR-GNR structure, the TC is reduced by about $87 \%$, indicating the strong hybridization effect between resonant and propagating modes. Such hybridization can reduce both the phonon group velocities and the phonon relaxation times, particularly at low frequencies [3, 12, 28], in contrast to the purely scattering effect $[2,13]$. Therefore, the TC of PR-GNR at low frequencies is significantly reduced compared to GNR, as can be seen from Fig. 4.

Similarly, the TC values for DR-GNR, AR-GNR, DAR-GNR, HR-GNR-2m, and HR-GNR-4m were calculated to be $490 \pm 41,360 \pm 9,562 \pm 27,468 \pm 4$, and $520 \pm 3 \mathrm{~W} / \mathrm{mK}$, respectively, as shown in Fig. 3. They are all larger than the TC of PR-GNR. From Fig. 4, we also see that the enhancement of TC in DR-GNR compared to PR-GNR is contributed by both low- and high-frequency phonons, while the enhancement of TC in AR-GNR and HR-GNR comes from the high-frequency phonons only. The abnormal increase of TC in all these modified resonant structures compared to PR-GNR is not expected from the phonon scattering mechanism, but can be explained in terms of the phonon resonance mechanism, as discussed below.

\section{DISCUSSION}

As noted above, the reduction of TC in PR-GNR compared to pristine GNR is due to the resonant hybridization between the resonant modes from the pillars and the propagating modes in the base GNR. The enhancement of TC in the various imperfect resonant structures is then due to the weakening (or decoupling) of the resonant hybridization caused by the imperfections. It turns out that the weakening of the resonant hybridization by interface 
(a)

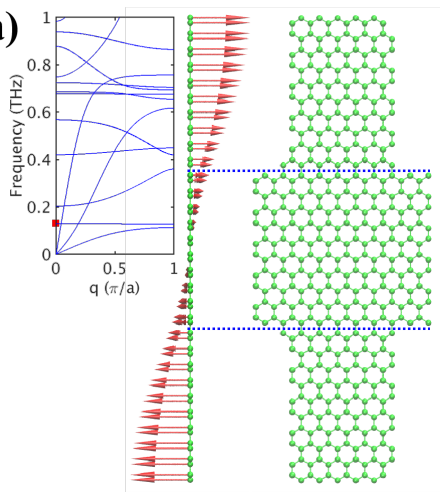

(b)

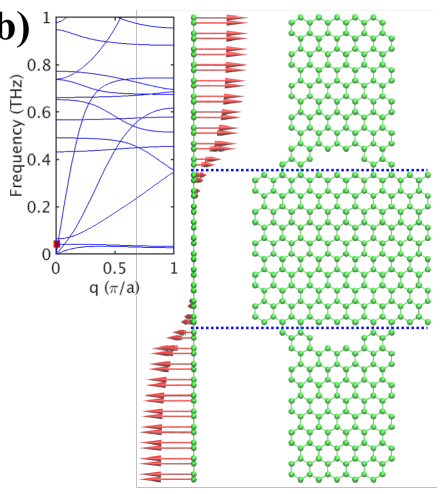

(c)

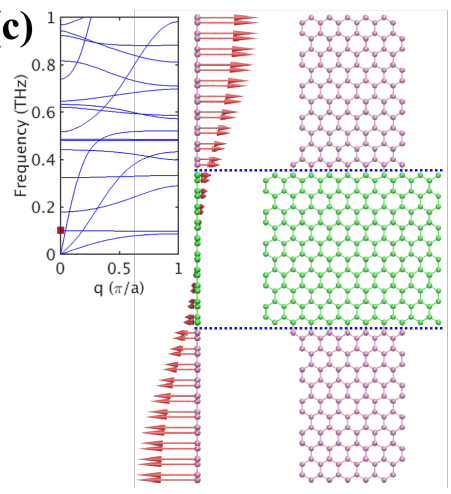

FIG. 5. Phonon dispersion at low frequencies, eigenvector of the first resonant mode (the arrows), and the corresponding structure for (a) PR-GNR, (b) DR-GNR, and (c) HR-GNR-2m. The frequency of the first resonant mode is marked as a red dot in each phonon dispersion diagram. The dashed lines are guides to the eye (see text). To make the LD calculation affordable, here we reduced the height of pillars to $2 \mathrm{~nm}$. This does not affect the conclusions here.

defect (in DR-GNR) or mass mismatch (in HR-GNR) at the interface between the pillars and the base material is mainly due to harmonic phonon properties, while the weakening of the resonant hybridization by alloy disorder (in AR-GNR) is mainly due to anharmonic phonon properties, as we explore in turn below.

A. Weakening of resonant hybridization by interface defect and mass mismatch due to harmonic effects

In order to understand the role of phonon hybridization in the heterostructures here, we used harmonic LD to calculate the phonon dispersions in the quasi-onedimensional PR-GNR, DR-GNR, and AR-GNR. Figure 5 shows the low-frequency parts, where the resonant modes with relatively flat phonon bands $[11,13]$ can be clearly seen. The first resonant frequency is at about $0.13 \mathrm{THz}$ in PR-GNR, which is reduced to about $0.043 \mathrm{THz}$ in DRGNR due to the weakening of the mechanical coupling strength between pillars and the base GNR by the vacancy defects at the interfaces. In HR-GNR, all the resonant frequencies are reduced due to the increased atomic mass in the resonant pillars, with the first resonant frequency reduced to about $0.10 \mathrm{THz}$.

The eigenvector for the first resonant frequency is also illustrated in Fig. 5 for each resonant structure, which corresponds to a rotation of the pillars around the base GNR. In PR-GNR, the magnitude of the eigenvector component decreases rather smoothly from the top of a pillar to the center of the base GNR. Therefore, this resonant mode penetrates deeply into the base GNR and will strongly couple to the propagating modes in the base GNR. In HR-GNR, and particularly in DR-GNR, the change of the magnitude of the eigenvector component is less continuous, leading to reduced penetration of resonant modes, which weakens the coupling between res- onant and propagating modes and abnormally enhances the TC.
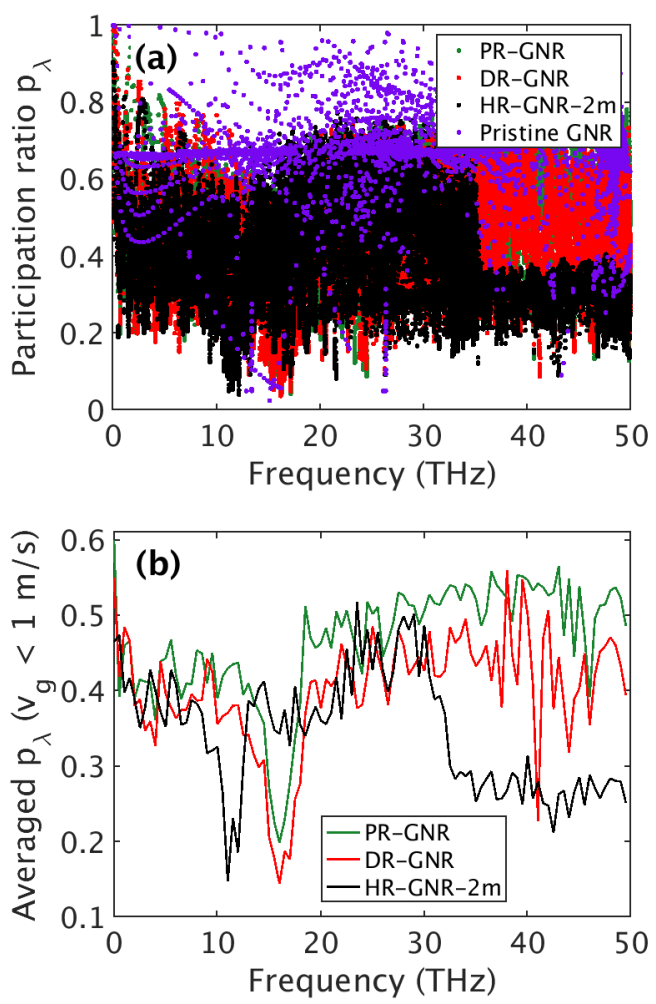

FIG. 6. (a) Participation ratio in pristine GNR, PR-GNR, DR-GNR and HR-GNR-2m. (b) Averaged participation ratio for modes with group velocity smaller than $1 \mathrm{~m} / \mathrm{s}$ in the resonant structures. Suggestion: use different markers in both (a) and (b), not only relying on different colors.

The weakening of the resonant hybridization in DRGNR and HR-GNR can also be understood in terms of phonon localization. A stronger phonon localization cor- 
responds to a smaller phonon participation ratio $(\mathrm{PR})$ defined as [29, 30]:

$$
p_{\lambda}=\left[N \sum_{i=1}^{N}\left(\sum_{\alpha} u_{i \alpha, \lambda}^{*} u_{i \alpha, \lambda}\right)^{2}\right]^{-1}
$$

Here, $u_{i \alpha, \lambda}$ is the component of the vibrational eigenvector of atom $i$ in direction $\alpha$ for mode $\lambda$ and $N$ is the number of atoms in the unit cell.

Figure 6(a) shows the PR values for all the modes in pristine GNR, PR-GNR, DR-GNR, and HR-GNR. The $P R$ values in the resonant structures are much smaller than those in pristine GNR, in accordance with the fact that a reduced participation ratio, hence an enhanced localization, usually corresponds to a reduced TC [31-33]. However, this rule is only valid for propagating modes. For resonant (non-propagating) modes, a reduction of PR (enhancement of phonon localization) should lead to an enhancement of TC as the enhanced localization of the resonant modes will reduce the resonant hybridization between the resonant and propagating modes. Figure 6(b) shows the averaged PR values over the modes with group velocity $v_{\mathrm{g}}<1 \mathrm{~m} / \mathrm{s}$ in the resonant structures, which are almost contributed by the resonant modes solely. It can be seen that the PR values for the nonpropagating resonant modes in DR-GNR and HR-GNR are indeed smaller than those in PR-GNR.

B. Weakening of resonant hybridization by alloy disorder due to anharmonic effects

Different from DR-GNR and HR-GNR, the weakening of resonant hybridization in AR-GNR is not due to the blocking of the penetration of the resonant modes into the base GNR, but due to the phonon-disorder scattering within the alloyed pillars. To understand this, we first note that when the intrinsic phonon MFP is much larger than the pillar height for all frequencies, phonons can hardly be scattered inside the pillars and regular phonon resonance can be formed whenever the phase difference of the incoming and outgoing (reflected) phonons is $(2 n+$ 1) $\pi$, where $n$ is an integer. This is the case for PR-GNR, where the intrinsic phonon MFP inside the pillars (which can be considered as pristine GNRs) are larger than the pillar height $(10.2 \mathrm{~nm})$ for all frequencies, as shown in Fig. 7.

From Fig. 7, we also see that when the pillars are alloyed as in AR-GNR, the phonon MFP inside the pillars is reduced due to phonon-disorder scattering, which is smaller than the pillar height for $\omega / 2 \pi>18 \mathrm{THz}$. As a result, the phase relation of the original resonant modes with $\omega / 2 \pi>18 \mathrm{THz}$ will be changed, thus affecting the formation of those resonant modes. The break of the original phase relation only means that the original regular resonant modes are destroyed. However, it can still create random resonant modes as some of the scattered phonons still satisfy the phase relation. Such a random resonant effect is weaker than the regular resonant one as it cannot be generated for all wavelengths at a specific frequency, i.e., the resonant band cannot be formed in the entire Brillouin zone [2]. The weakening of the resonant modes for $\omega / 2 \pi>18 \mathrm{THz}$ agrees with the enhanced TC in AR-GNR compared to PR-GNR for the high frequency phonons only as shown in Fig. 4.



FIG. 7. Phonon mean free path of the pristine and $50 \%$ alloyed GNR with width of $4.0 \mathrm{~nm}$, calculated according to Eq. (4). The red dashed line corresponds to the height of the resonant pillars $(10.2 \mathrm{~nm})$. Better to use one solid line and one dotted line.

\section{SUMMARY AND CONCLUSIONS}

In summary, we have studied the effects of imperfections on phonon resonance in resonant nanostructures based on GNR, using molecular dynamics simulations and lattice dynamics analysis. It is demonstrated that in contrast to the phonon scattering picture of TC reduction by imperfection, introducing imperfections to the resonant structures, such as vacancy defects at the interfaces, mass mismatch between the pillars and the base material, and alloy disorder in the pillars can all weaken the resonant hybridization and abnormally increase the TC. Based on harmonic lattice dynamics analyses, we have clarified that both vacancy defects and mass mismatch can reduce the penetration of the resonant modes from the pillars into the base material. On the other hand, alloy disorder in the pillars can scatter the phonons inside the pillars, which turns regular resonance into random resonance with weaker hybridization. The large enhancement of TC in resonant structures by imperfections could explain the weak reduction of $\mathrm{TC}$ by phonon resonance in the aluminum-pillared silicon membranes as observed in the recent experiments [34], where various imperfections are expected within the pillars and at the interfaces between the pillars and the base material. 


\section{ACKNOWLEDGEMENTS}

This work was supported by the National Natural Science Foundation of China (NSFC) (No. 11804242), Collaborative Innovation Center of Suzhou Nano Science \& Technology (Nano-CIC), the Priority Academic Program Development of Jiangsu Higher Education Institutions
(PAPD), the 111 Project, and Joint International Research Laboratory of Carbon-Based Functional Materials and Devices. ZF and TA-N acknowledge the supports from the NSFC (No. 11974059) and in part by the Academy of Finland QTF Centre of Excellence program (Project 312298).
[1] J. Maire, R. Anufriev, T. Hori, J. Shiomi, S. Volz, and M. Nomura, Scientific Reports 8, 4452 (2018).

[2] S. Xiong, D. Selli, S. Neogi, and D. Donadio, Phys. Rev. B 95, 180301 (2017).

[3] H. Honarvar and M. I. Hussein, Phys. Rev. B 97, 195413 (2018).

[4] M. I. Hussein, C.-N. Tsai, and H. Honarvar, Advanced Functional Materials 30, 1906718 (2020).

[5] H. Honarvar and M. I. Hussein, Phys. Rev. B 93, 081412 (2016).

[6] A. Giri and P. E. Hopkins, Journal of Applied Physics 125, 205102 (2019).

[7] D. Ma, H. Ding, H. Meng, L. Feng, Y. Wu, J. Shiomi, and N. Yang, Phys. Rev. B 94, 165434 (2016).

[8] A. Giri and P. E. Hopkins, Phys. Rev. B 98, 045421 (2018).

[9] H. Zhang, B. Sun, S. Hu, H. Wang, Y. Cheng, S. Xiong, S. Volz, and Y. Ni, Phys. Rev. B 101, 205418 (2020).

[10] X.-K. Chen, J. Liu, Z.-X. Xie, Y. Zhang, Y.-X. Deng, and K.-Q. Chen, Applied Physics Letters 113, 121906 (2018).

[11] B. L. Davis and M. I. Hussein, Phys. Rev. Lett. 112 , 055505 (2014).

[12] S. Neogi and D. Donadio, Phys. Rev. Applied 14, 024004 (2020).

[13] S. Xiong, K. Sääskilahti, Y. A. Kosevich, H. Han, D. Donadio, and S. Volz, Phys. Rev. Lett. 117, 025503 (2016).

[14] R. Anufriev and M. Nomura, Science and Technology of Advanced Materials 19, 863 (2018), pMID: 30479674.

[15] S. Volz, J. Ordonez-Miranda, A. Shchepetov, M. Prunnila, J. Ahopelto, T. Pezeril, G. Vaudel, V. Gusev, P. Ruello, M. Weig, Eva M.and Schubert, M. Hettich, M. Grossman, T. Dekorsy, F. Alzina, B. Graczykowski, E. Chavez-Angel, J. S. Reparaz, M. R. Wagner, C. M. Sotomayor-Torres, S. Xiong, S. Neogi, and D. Donadio, The European Physical Journal B 89, 15 (2016).

[16] J. Recatala-Gomez, A. Suwardi, I. Nandhakumar, A. Abutaha, and K. Hippalgaonkar, ACS Applied Energy Materials 3, 2240 (2020).

[17] N. Li, J. Ren, L. Wang, G. Zhang, P. Hänggi, and B. Li, Rev. Mod. Phys. 84, 1045 (2012).
[18] Z. Fan, W. Chen, V. Vierimaa, and A. Harju, Computer Physics Communications 218, 10 (2017).

[19] Z. Fan and A. Gabourie, "GPUMD-v2.5.1," (2020).

[20] L. Lindsay and D. A. Broido, Phys. Rev. B 81, 205441 (2010).

[21] D. J. Evans, Physics Letters A 91, 457 (1982).

[22] Z. Fan, H. Dong, A. Harju, and T. Ala-Nissila, Phys. Rev. B 99, 064308 (2019).

[23] K. Sääskilahti, J. Oksanen, J. Tulkki, and S. Volz, Phys. Rev. B 90, 134312 (2014).

[24] K. Sääskilahti, J. Oksanen, S. Volz, and J. Tulkki, Phys. Rev. B 91, 115426 (2015).

[25] Z. Fan, L. F. C. Pereira, P. Hirvonen, M. M. Ervasti, K. R. Elder, D. Donadio, T. Ala-Nissila, and A. Harju, Phys. Rev. B 95, 144309 (2017).

[26] A. J. Gabourie, Z. Fan, T. Ala-Nissila, and E. Pop, "Spectral Decomposition of Thermal Conductivity: Comparing Velocity Decomposition Methods in Homogeneous Molecular Dynamics Simulations," ArXiv:2102.03691 [cond-mat.mtrl-sci].

[27] Z. Li, S. Xiong, C. Sievers, Y. Hu, Z. Fan, N. Wei, H. Bao, S. Chen, D. Donadio, and T. Ala-Nissila, The Journal of Chemical Physics 151, 234105 (2019).

[28] H. Wang, Y. Cheng, M. Nomura, S. Volz, D. Donadio, X. Zhang, and S. Xiong, Phys. Rev. B 103, 085414 (2021).

[29] P. K. Schelling, S. R. Phillpot, and P. Keblinski, Phys. Rev. B 65, 144306 (2002).

[30] A. Bodapati, P. K. Schelling, S. R. Phillpot, and P. Keblinski, Phys. Rev. B 74, 245207 (2006).

[31] S. Pailhès, H. Euchner, V. M. Giordano, R. Debord, A. Assy, S. Gomès, A. Bosak, D. Machon, S. Paschen, and M. de Boissieu, Phys. Rev. Lett. 113, 025506 (2014).

[32] L. Yang, J. Chen, N. Yang, and B. Li, International Journal of Heat and Mass Transfer 91, 428 (2015).

[33] M. Hu, K. P. Giapis, J. V. Goicochea, X. Zhang, and D. Poulikakos, Nano Letters 11, 618 (2011).

[34] X. Huang, D. Ohori, R. Yanagisawa, R. Anufriev, S. Samukawa, and M. Nomura, ACS Applied Materials \& Interfaces 12, 25478 (2020). 